\title{
Transforming Medical Education
}

\section{Academia Meets Innovation}

Taylor Novice, BS ${ }^{1}$; Adish Parikh, BS ${ }^{1}$; Rahul Iyengar, BSE

A s the medical landscape changes, future physicians will face new demands. The pressure to combat increasing health care costs places a more poignant focus on efficiency and cost effectiveness than ever before. ${ }^{1}$ Educating medical students, as well as current physicians, about innovation and interdisciplinary collaborations may provide a way to tackle these challenges by creating cost-cutting protocols and technologies.

Dr Victor Dzau, president and CEO of Duke University Health System, urges academic health centers (AHCS) to emphasize the importance of innovation. Through his role, Dr Dzau has experienced how limited funding has placed new demands on AHCS. Because of these demands, he recommends AHCs seek new research and other collaborations with "diverse partners - including nonmedical university disciplines, industry, and businesses." He pushes further and directly calls for action: the health care environment must not just "allow" innovation but "actively [encourage] it to happen anywhere at every level."2
In response to these types of recommendations, $\mathrm{AHC}$ leaders have pushed for the reinvention of medical education and training. ${ }^{2}$ They have shortened preclinical years and have focused on adding extra time for students to pursue individual interests. Recently, we have seen shifts in medical education to incorporate entrepreneurial opportunities for students. For example, the University of Michigan Medical School has created an academic track in innovation and entrepreneurship; Harvard Medical School has partnered with Harvard Business school to create a health care innovation acceleration program.

Still, we believe medical students are not fully prepared to engage with technological developments and form public-private partnerships. Current curricula focus on the intricacies of past medical science, best practices, and clinical research. While these topics are undoubtedly key to a strong education, are medical students, as future doctors, being deliberately trained to navigate demands on physicians to be forward thinkers, apply cost-effective 
measures, and sense trends in the field? Does the current education system sufficiently empower tomorrow's physicians to handle the inevitable influences from the government and private sector? Dr Robert Pearl, a surgeon, CEO of Permanente Medical Group, and a Stanford Business School professor, surmises that while medical education focuses on a "right way" to provide medical care full of definitive answers, it may not provide the "skill set and [ . . . ] creativity" that future physicians will require for the growing collaborations in health care. ${ }^{2}$ He commends Bio-X, a Stanford University interdisciplinary initiative; this program has reported impressive achievements in translating basic research to patient care and commercialization, particularly in medical devices. ${ }^{3}$

We therefore call for AHCs to implement a more systematic and direct integration of developments in technology, private industry, and public health into the medical school curriculum. Through integration of the following, medical education can prepare physicians with the skills to navigate the new financial and technological challenges of our health care system.

First, academic curricula should place increased emphasis on presenting areas for innovation in the medical field to students. Since students have limited clinical exposure in their early years of medical school, many have found it difficult to pinpoint health care inefficiencies. If professors introduced students early on to gaps in care and technology, they may encourage students to practice creating solutions to problems they would not have known about otherwise. For example, when lecturers address image interpretation, they could discuss the increasingly dominant role artificial intelligence is playing in shaping radiology as well as any challenges the technology is facing. ${ }^{4}$

Second, in addition to classroom lectures, we see value in challenging trainees to work together to solve real-world clinical problems. For example, AHCs and medical schools could sponsor case-based competitions for residents and medical students with incentives for participation and performance. These competitions could focus on areas for quality improvement, efficient care delivery, and technology. Many business schools now focus on case study and analysis because this method of critical thinking motivates students to solve complex problems in ways that are not covered in lectures. ${ }^{5}$

Third, medical schools should actively foster connections to private industry. Curricula should include real-world experiences, such as internships with biomedical engineering firms and pharmaceutical companies, or courses on how to translate novel research into medical solutions. By working with business, medical trainees can gain valuable skills that can ultimately benefit $\mathrm{AHCs}^{\prime}$ long-term goals.

A smaller scale implementation of an innovation curriculum has had success at the University of Michigan. In the innovation and entrepreneurship pathway, students can pair with a mentor who introduces them to a specific clinical problem and helps them to develop a solution. Some of these projects are currently applying for funding, and others have entered prototype stages. Separately, the second year medical students have organized a Shark Tank competition in which teams of students developed a solution to a clinical problem; formed partnerships with technical, legal, and business experts; and pitched to a panel of surgeons. Ideas ranged from G-tubes with low dislodgement rates to a "smart toilet" able to detect urine glucose levels. While many teams ran into technical feasibility and intellectual property issues, these roadblocks pushed them to pivot and adjust their ideas. Participants emphasized that this process taught them new approaches to addressing problems that focus on flexibility and willingness to fail and try again. Encouraging medical trainees to think this way can empower them to develop these nonclinical skills essential for innovative solutions.

As second-year medical students, we have already benefitted from opportunities in medical device innovation. During the summer between first and second year of medical school, 
in addition to working on academic health care research, we participated in a part-time internship at IncWell, a health care venture capital firm based in Birmingham, Michigan. Under the mentorship of Dr Reda Jaber, an MD/MBA from University of Michigan and a partner at IncWell, we gained exposure to cutting edge health care trends, such as one-drop diagnostics and 3-D biological printing. We were part of multidisciplinary teams that thoroughly evaluated these health care start-ups' needs assessment, product feasibility, and business model. Through these interactions, we developed a broader perspective on how stakeholders contribute to both the success and failure of the health care system. This perspective will help us work more effectively with engineers, pharmaceutical companies, biotech firms, and government agencies to address pressing health care issues.

Our interaction with owners and investors will allow us to approach our second year of medical studies with a critical eye for ways to introduce cost-effective technologies with the potential to improve patient care. Empowering medical students to broaden their perspective today can aid in the development of tomorrow's physician-leaders capable of confidently addressing the most prominent health care obstacles.

\section{References}

1. Dzau VJ, et al. Transforming academic health centers for an uncertain future. N Eng/ J Med. 2013;369(11):991-993.

2. Dzau VJ, et al. Fostering innovation in medicine and health care: what must academic health centers do? Acad Med. 2013;88(10):1424-1429.

3. Pearl R. "What doctors can learn from business leaders." Forbes, August 1, 2013. Web.

4. Shiraishi J, et al. Computer-aided diagnosis and artificial intelligence in clinical imaging. Semin Nucl Med. 2011;41(6): 449-462.

5. Levy F. Harvard Business School has the market cornered on case studies. BloombergBusinessweek, April 9, 2015. Bloomberg, www.bloomberg.com/ news/articles/2015-04-09/harvard-s-case-study -monopoly. 